\title{
A gestão da enfermagem e a implantação das metas de segurança do paciente em uma unidade de emergência pública
}

\author{
Nursing management and the implementation of patient safety goals in a public \\ emergency unit
}
Gestión de enfermería e implementación de objetivos de seguridad del paciente en una unidad de emergencia pública

Daniella Ramalhoto Ramos ${ }^{1}$, Renato Barbosa Japiassu*, Chennyfer Dobbins Abi Rached².

\section{RESUMO}

Objetivo: Analisar as produções científicas sobre a gestão da enfermagem na implantação de metas de segurança ao paciente em unidades de emergência pública. Revisão bibliográfica: O gerenciamento da segurança do paciente no departamento de emergência inclui a identificação e priorização de riscos, o desenho e a aplicação de planos de melhoria e a avaliação dos resultados de sua implementação. Quando o trabalho é iniciado nessa área, é provável que os dados e resultados próprios (ou de ambientes próximos) ainda não estejam disponíveis ou sejam escassos. Assim, o papel do líder no processo de iniciação e implantação da cultura de segurança, propõe-se que este deverá conduzir os liderados por meio da comunicação efetiva, da promoção do desenvolvimento profissional, através de capacitações, do incentivo às práticas seguras e da realização do feedback. Considerações finais: A segurança do paciente implica fornecer cuidados de saúde livres de danos que possam ser evitados, o que implica o desenvolvimento de estratégias que contribuam para reduzir as chances de falhas nos processos, bem como os erros humanos. Dessa forma, é necessário ampliar as discussões sobre o propósito do trabalho nos serviços de emergência visando a segurança do paciente, para que o trabalhador possa assumir uma postura crítica.

Palavras-chave: Gestão hospitalar, Segurança do paciente, Ciência da implementação.

\begin{abstract}
Objective: Analyze scientific production on nursing management in the implementation of patient safety goals in public emergency units. Bibliographic review: Managing patient safety in the emergency department includes identifying and prioritizing risks, designing, and applying improvement plans, and evaluating the results of their implementation. When work is initiated in this area, it is likely that data and results of its own (or from nearby environments) are not yet available or scarce. Thus, the role of the leader in the process of initiation and implementation of the safety culture, it is proposed that this should lead the followers through effective communication, the promotion of professional development, through training, the encouragement of safe practices and the realization of feedback. Final considerations: Patient safety implies providing health carefree of harm that can be avoided, which implies the development of strategies that contribute to reduce the chances of failures in the processes, as well as human errors. Thus, it is necessary to expand the discussions on the purpose of work in emergency services aiming at patient safety, so that the worker can assume a critical posture.
\end{abstract}

Keywords: Hospital management, Patient safety, Implementation science.

${ }^{1}$ Faculdade Unyleya, Brasília - DF. *E-mail: renatojapiassu@gmail.com

2 Universidade de São Paulo (USP), São Paulo - SP. 


\section{RESUMEN}

Objetivo: Analizar la producción científica sobre la gestión de enfermería en la implementación de las metas de seguridad del paciente en las unidades públicas de emergencia. Revisión bibliográfica: La gestión de la seguridad del paciente en el servicio de urgencias incluye identificar y priorizar riesgos, diseñar y aplicar planes de mejora y evaluar los resultados de su implementación. Cuando se inicie el trabajo en esta área, es probable que los datos y resultados propios (o de entornos cercanos) aún no estén disponibles o sean escasos. Así, el rol del líder en el proceso de iniciación e implementación de la cultura de seguridad se propone que esta debe conducir a los seguidores a través de la comunicación efectiva, la promoción del desarrollo profesional, a través de la capacitación, el fomento de prácticas seguras y la realización de retroalimentación. Consideraciones finales: La seguridad del paciente implica brindar una atención de salud libre de daños evitables, lo que implica el desarrollo de estrategias que contribuyan a reducir las posibilidades de fallas en los procesos, así como errores humanos. Por tanto, es necesario ampliar las discusiones sobre la finalidad del trabajo en los servicios de urgencias con miras a la seguridad del paciente, para que el trabajador asuma una postura crítica.

Palabras clave: Gestión hospitalaria, Seguridad del paciente, Ciencia de implementación.

\section{INTRODUÇÃO}

As unidades hospitalares de atenção a emergências integram o componente hospitalar do sistema de atenção. $O$ objetivo do trabalho das equipes de saúde dessas unidades é atender pacientes que chegam em estado grave, aceitar os urgentes e prosseguir para encaminhá-los para ambulatório da rede básica ou especializada, tratando de garantir o acesso e recepção em serviços de saúde, de acordo com a complexidade tecnológica, que deve ser organizada de forma regionalizada, hierarquizada e regulada (ARAÚJO MO, 2014; ARAÚJO LMA, 2017).

Alguns estudos realizados no Reino Unido relataram que o envolvimento direto dos diretores executivos em questões gerenciais da instituição hospitalar melhorou consideravelmente a segurança e a qualidade da assistência prestada. Foi mostrado que a presença frequente de gestores/gerentes em unidades assistenciais hospitalares promove maior confiança entre as equipes, pois eles estão próximos dos problemas e conseguem identificar prontamente. A partir desta presença e escuta, puderam influenciar nos processos internos e alocar recursos para as áreas prioritárias (BAMPI R, et al., 2017).

A equipe de enfermagem tem um papel importante na prevenção de incidentes, não só por agregar a maior quantidade de profissionais, mas também pelo contato direto com os pacientes, percebendo as reais necessidades e antevendo situações de cada usuário. Porém, não somente a enfermagem é responsável pela assistência, mas toda a equipe multidisciplinar tem o seu papel na melhoria do processo de trabalho e no compromisso com os resultados necessários para a segurança do paciente no âmbito hospitalar. A falta de gerenciamento eficaz dificulta a realização da assistência, desestabiliza a equipe e pode induzir a erros, como os incidentes relacionados a prestação de cuidado (BAMPI R, et al., 2017).

A segurança do paciente envolve as ações promovidas pelas instituições de saúde, especialmente as unidades hospitalares, tendo como função reduzir, a um mínimo aceitável, o risco de dano desnecessário relacionado ao cuidado em saúde. Existem alguns serviços hospitalares para emergências que são organizados hierarquicamente que atendem a esse modelo de atendimento (ARAÚJO MO, 2014).

Outro conceito para entendimento da segurança do paciente que poderá ser compreendida como redução, a um mínimo aceitável, do risco de dano desnecessário no cuidado a saúde das pessoas. Alguns estudos estimam que a ocorrência de incidentes relacionados à assistência à saúde, e, em particular, os eventos adversos ocorridos, afete cerca de $4 \%$ a $16 \%$ de pacientes que estão hospitalizados em países desenvolvidos, o que sensibilizou os sistemas de saúde em todo o mundo a melhorar a segurança do paciente (BRANDÃO MGSA, et al., 2018). A Organização Mundial da Saúde (OMS) também tem a sua definição sobre a segurança do paciente, que é a redução do risco de danos desnecessários a um mínimo aceitável, associado a assistência em saúde (FERREIRA BEM, et al., 2021). 
A segurança do paciente é influenciada, quando os profissionais da área de saúde não estão devidamente treinados, capacitados ou falta de conhecimento relacionado a esse tema, podendo ocorrer alguma situação de dano na assistência em saúde, as quais refletem diretamente na qualidade de vida dos clientes, provocando consequências desagradáveis tanto para os pacientes como para os profissionais e para a organização hospitalar (SILVA AT, et al., 2016).

Os profissionais de enfermagem são responsáveis por grande parte das ações assistenciais e, portanto, encontram-se em posição privilegiada para reduzir a possibilidade de incidentes que atinjam o paciente, além de detectar as complicações precocemente e realizar as condutas necessárias para minimizar os danos que possam ocorrer. Assim, o assunto da segurança do paciente torna-se essencial (SILVA FS, 2018).

Os riscos para incidentes estão somente associados ao erro humano dos profissionais de enfermagem, mas deve-se levar em consideração alguns outros fatores, como as condições de trabalho, os aspectos estruturais e a complexidade das atividades desenvolvidas. As situações que levam ao risco dos incidentes podem incluir o avanço tecnológico com deficiente aperfeiçoamento dos recursos humanos, a desmotivação, as falhas na aplicação da Sistematização da Assistência de Enfermagem (SAE), na delegação de cuidados sem supervisão adequada e a sobrecarga de serviço (BRANDÃO MGSA, et al., 2018).

Alguns estudos já evidenciaram incidentes causados aos pacientes pelos profissionais de enfermagem nas instituições de saúde no Brasil e no mundo. Com esses dados, o Ministério da Saúde (MS), o órgão do Poder Executivo Federal responsável pela organização e elaboração de planos e políticas públicas voltados para promoção, prevenção e assistência à saúde dos brasileiros, resolveu instituir, pela Portaria MS ํㅡ 529 , de 1 de abril de 2013, o Programa Nacional de Segurança do Paciente (PNSP), com o objetivo de implementar medidas assistenciais, educativas e programáticas e iniciativas voltadas à segurança do paciente em diferentes áreas da atenção, organização e gestão de serviços de saúde por meio da implantação da gestão de risco e de Núcleos de Segurança do Paciente (BEHRENS R, 2019; BRANDÃO MGSA, et al., 2018).

A relevância da PNSP para a enfermagem é que a temática sobre a segurança do paciente envolve toda a equipe de enfermagem na melhoria da qualidade da assistência prestada, pois ajudam os profissionais a reconhecer as causas e os efeitos à saúde do paciente, permitindo uma capacitação adequada com o emprego de roteiros e listas de verificação, sendo utilizados os protocolos para nortear uma melhor assistência e prevenir os incidentes nos serviços de saúde (FERREIRA BEM, et al., 2021).

Desse modo, é necessário compreender a relação entre os incidentes, as características dos cuidados de enfermagem à saúde e o aporte da rede hospitalar, podendo fornecer a esses profissionais alguns elementos importantes para a melhoria da assistência prestada. Embora os riscos relacionados aos cuidados de enfermagem estejam sendo abordados na literatura, é importante que se conheça os riscos de incidentes para elaborar estratégias de prevenção (BRANDÃO MGSA, et al., 2018).

Sendo assim, com base em tais aspectos, o objetivo deste artigo é analisar as produções científicas sobre a gestão da enfermagem na implantação de metas de segurança ao paciente em unidades de emergência pública.

\section{REVISÃO BIBLIOGRÁFICA}

Nas circunstâncias atuais, a urgência é quase definida mais pelo local onde é praticado do que pelo seu conteúdo formal: todo indivíduo que chega ou consulta em um hospital ou local de emergência primário é um caso de urgência. Às vezes, a urgência pode evoluir para uma emergência, e esta é uma situação crítica (SOUZA TH, 2014).

A emergência é um tipo de urgência vital, onde existe um risco presente imediato ou potencial a vida ou órgão do paciente, devido a um comprometimento das funções vitais (respiratória, cardíaca, neurológicas, dentre outras) ou sequelas graves permanentes de doenças anteriores (COSTA BHF, 2017).

Quando se fala em gestão de emergência, deve-se basear na existência de um sistema de emergência abrangente e de um centro de coordenação para emergências de saúde. O paciente que sofre um processo que gera emergência pode ter a melhoria da expectativa e qualidade de vida quando tem um sistema que 
garanta a sua atenção quando há uma intercorrência que necessite ser atendido rapidamente, no qual possa conseguir uma assistência em saúde, garantindo o atendimento necessário e resolvendo seu problema de saúde (CARVALHO LM, 2019).

Assim, é necessário que os enfermeiros repensem a sua prática profissional, pois, quando ele assume a sua função como o coordenador da assistência de enfermagem, fazendo implementações por meio de esquemas de planejamentos, garantindo o desenvolvimento das suas atividades básicas administrativas, assistenciais e de ensino, promovendo, assim, uma melhor organização do trabalho em equipe, passando a direcionar seus esforços em busca de um objetivo comum, prestando assistência de qualidade, atendendo as reais necessidades apresentadas pelos pacientes sob seus cuidados e zelando a segurança de seus pacientes (RORATO TJ, et al., 2021).

A segurança do paciente é considerada como uma forma de cuidado humanizado e prioridade na atenção à saúde, uma atividade cada vez mais complexa que acarreta riscos potenciais e na qual não há sistema capaz de garantir a ausência de efeitos adversos, uma vez que é uma atividade em que combinam os fatores inerentes ao sistema com as ações humanas, sendo a gestão de risco cada vez mais utilizada nos serviços de saúde (KOERICH C, 2019; SOARES VEA, 2019).

A gestão de riscos envolve a identificação, avaliação, análise, tratamento e a comunicação dos riscos, muito importante para melhorar a qualidade da assistência prestada, pois é durante os cuidados de enfermagem que há possibilidades de incidentes. As atuais pesquisas no cenário brasileiro mostram que a ocorrência desses incidentes de segurança são todos preveníveis nos serviços de saúde, desde que haja na instituição a educação continuada sobre a segurança do paciente (SCHMITT MD et al., 2020).

Os principais objetivos da segurança do paciente são aumentar a qualidade do atendimento, melhorar a segurança dos pacientes e dos profissionais de saúde no qual o recebem. A preocupação com a qualidade dos cuidados, com a segurança dos pacientes e com a confidencialidade das informações clínicas é geral nos sistemas de saúde e se reflete em diferentes normas legais e regulamentares, independentemente de sua forma organizacional e financeira, sendo necessário implementar as metas para a segurança do paciente nas instituições de saúde (KOERICH C, 2019; SOARES VEA, 2019).

As metas Internacionais de Segurança do Paciente são baseadas em ações específicas para melhorar a segurança do paciente, identificadas em políticas globais e apoiadas pelo registro do maior número de eventos adversos em atendimento da equipe de saúde. Por esse motivo, com a implementação de ações voltadas à garantia de processos de atendimento dos profissionais de saúde como prioridade, por meio das estratégias traçadas nas referidas metas, os eventos adversos serão significativamente reduzidos (SIMAN AG e BRITO MJM, 2016).

Estabelecer as diretrizes das metas Internacionais de Segurança do Paciente permite uniformizar as atividades do pessoal que participa da assistência de enfermagem ao usuário, criando uma metodologia operacional na qual é possível reduzir os riscos para ele. As responsabilidades da equipe de gerenciamento são: divulgar, avaliar, supervisionar e aconselhar a aplicação deste guia ao pessoal dos diferentes serviços de uma instituição; assegurar que as ações de segurança sejam realizadas dentro da estrutura de respeito absoluto pelos direitos humanos e responder às necessidades e expectativas dos pacientes e suas famílias; monitorar o fornecimento de insumos e uso racional, com base nas necessidades identificadas e prioridades estabelecidas, no âmbito da segurança do paciente (SILVA RFA e NASCIMENTO MAL, 2012).

Com o intuito de promover uma melhoria na assistência, as instituições de saúde estão padronizando os protocolos assistenciais, apresentando inovações tecnológicas e estruturais que incorporam as Metas Internacionais de Segurança do Paciente (MISP). O objetivo das MISP é obter padrões elevados de qualidade na assistência, por meio de iniciativas que propiciem o seu desenvolvimento, tais como aquelas direcionadas a competências profissionais, mudanças comportamentais, mobilização gerencial, inovações estruturais e tecnológicas, além da melhoria permanente e contínua do atendimento (ROSA ERS, et al., 2017). As metas estabelecidas abaixo devem ser levadas a sério para que se consiga a segurança do paciente. Por isso, é importante que o gestor esteja acompanhando o cumprimento de todas as seguintes metas: 
Meta 1: identificar corretamente os pacientes - o pessoal da instituição deve evitar erros de identificação de pacientes, considerando que pode ocorrer em todos os processos de atendimento em que muitos profissionais de saúde, pois usam três identificadores completos, nome do paciente, data de nascimento e número de inscrição antes da realização de procedimentos médicos, cirúrgicos, técnicos ou administrativos, de forma confiável e inequívoca nas diferentes áreas da saúde. O objetivo é identificar com segurança os pacientes para evitar erros que envolvam o paciente errado (FRANCISCATTO L, et al., 2011; INSTITUTO BRASILEIRO PARA A SEGURANÇA DO PACIENTE, 2018; OLIVEIRA JLC, et al., 2017; ROSA ERS, et al., 2017; VIANA ALVES EA, 2013).

Meta 2: melhorar a comunicação entre a equipe de saúde - a equipe deve prevenir erros que envolvem a comunicação mais propensa a erros, e utiliza o processo de ouvir, escrever, ler e confirmar a comunicação verbal de indicações e/ou em emergências que possam ocorrer durante os processos de cuidado no ambiente: áreas de internação, emergências, terapia intensiva, queimaduras e centro cirúrgico. $O$ objetivo é evitar erros que envolvam comunicações mais propensas a: pedidos e/ou informações sobre resultados críticos de laboratório e gabinete, seja ela verbal ou por telefone (FRANCISCATTO L, et al., 2011; INSTITUTO BRASILEIRO PARA A SEGURANÇA DO PACIENTE, 2018; OLIVEIRA JLC, et al., 2017; ROSA ERS, et al., 2017; VIANA ALVES EA, 2013).

Meta 3: melhorar a segurança na prescrição, no uso e na administração de medicamentos - eles devem ser identificados com alerta visual (círculo vermelho), separado do resto das drogas e dupla verificação que é realizada nos processos de preparação e ministração em áreas hospitalares de ortopedia, reabilitação, salas de cirurgia, unidade de terapia intensiva, queimaduras, ambulatório de ortopedia e imagiologia. Os objetivos são evitar a administração errônea de: eletrólitos, concentrados, quimioterápicos, radiofármacos, insulinas, anticoagulantes parenterais (FRANCISCATTO L, et al., 2011; INSTITUTO BRASILEIRO PARA A SEGURANÇA DO PACIENTE, 2018; OLIVEIRA JLC, et al., 2017; ROSA ERS, et al., 2017; VIANA ALVES EA, 2013).

Meta 4: assegurar cirurgia em local de intervenção, procedimento e paciente correto - a equipe deve garantir o cumprimento do protocolo universal antes de realizar os procedimentos cirúrgicos dentro e fora da sala de cirurgia. E reunir a confirmação das informações no período imediatamente antes do início da administração de sangue, componentes sanguíneos e execução de procedimentos invasivos nas áreas de tempo de assistência médica/cirúrgica: hospitalização, sala de operações, unidade de terapia intensiva, queimaduras, urgências, consulta Oftalmologia Externa, Otorrinolaringologia, Imagem e Emergência. Os objetivos são: evitar procedimentos cirúrgicos incorretos no lugar errado e a pessoa errada que são executados dentro e fora da sala de cirurgia; executar o procedimento correto, para a pessoa certa na hora certa para atender, antes da administração de sangue, componentes sanguíneos e execução de procedimentos invasivos em áreas de cuidados de saúde (FRANCISCATTO L, et al., 2011; INSTITUTO BRASILEIRO PARA A SEGURANÇA DO PACIENTE, 2018; OLIVEIRA JLC, et al., 2017; ROSA ERS, et al., 2017; VIANA ALVES EA, 2013).

Meta 5: higienizar as mãos para evitar infecções - a equipe deve reduzir o risco de infecções associadas aos cuidados de saúde e cumprir o protocolo de Higiene das Mãos com base nas diretrizes estabelecidas pela Organização Mundial da Saúde (OMS), cumprir o procedimento quinquenal e aplicar as medidas. Doenças para a prevenção de infecções em todas as áreas da saúde da Reabilitação, Ortopedia, Otorrinolaringologia, Oftalmologia, Medicina do Esporte, Audiologia, Fonoaudiologia e Linguística, Consulta Externa, Salas de Cirurgia, Unidade de Terapia Intensiva, Serviços Auxiliares de Diagnóstico, Queimaduras e Urgências. O objetivo é prevenir, reduzir e avaliar o risco de infecções associadas aos cuidados de saúde através da implementação adequada do protocolo de higiene das mãos (FRANCISCATTO L, et al., 2011; INSTITUTO BRASILEIRO PARA A SEGURANÇA DO PACIENTE, 2018; OLIVEIRA JLC, et al., 2017; ROSA ERS, et al., 2017; VIANA ALVES EA, 2013).

Meta 6: reduzir o risco de dano ao paciente causado por quedas - a equipe deve avaliar o risco de quedas a partir da avaliação inicial dos pacientes, reavaliar o risco de acordo com as mudanças no estado dos pacientes, reduzir o risco de quedas e cumprir o Protocolo de Prevenção de Quedas em pacientes 
Hospitalizado, nas áreas de saúde onde é prestado atendimento médico/cirúrgico. O objetivo é prevenir e reduzir as quedas de risco de pacientes hospitalizados (FRANCISCATTO L, et al., 2011; INSTITUTO BRASILEIRO PARA A SEGURANÇA DO PACIENTE, 2018; OLIVEIRA JLC, et al., 2017; ROSA ERS, et al., 2017; VIANA ALVES EA, 2013).

O gerenciamento da segurança do paciente no departamento de emergência inclui a identificação e priorização de riscos, o desenho e a aplicação de planos de melhoria e a avaliação dos resultados de sua implementação. Quando o trabalho é iniciado nessa área, é provável que os dados e resultados próprios (ou de ambientes próximos) ainda não estejam disponíveis ou sejam escassos. Em qualquer caso, as contribuições concretas oferecidas por várias organizações significativas no campo do Segurança do paciente para priorizar as ações de análise e melhoria em uma instituição particular são sempre muito úteis (CALDAS BN, 2017).

O objetivo da implantação das estratégias de segurança do paciente em uma emergência pública é reduzir os riscos desnecessários decorrentes da assistência à saúde. É promover melhorias específicas na segurança do paciente. Os objetivos destacam as áreas problemáticas dentro do atendimento de enfermagem e descrevem soluções acordadas para esses problemas, com base em evidências e conhecimento especializado. Reconhecendo que um projeto sólido do sistema é fundamental para a prestação de cuidados de enfermagem seguros e de alta qualidade, os objetivos geralmente se concentram em soluções que cobrem todo o sistema, sempre que possível (CALDAS BN, 2017).

Para que as emergências das organizações hospitalares obtenham sucesso durante o processo de implantação de tais estratégias, é essencial o fortalecimento de uma Cultura de Segurança do Paciente (CSP) (CALDAS BN, 2017). A CSP é considerada um importante componente para os serviços de saúde que favorece a sua implementação, com práticas seguras e diminuição de incidentes de segurança. Esse termo, cultura de segurança, foi utilizado inicialmente pela International Atomic Energy Agency (IAEA), quando foi publicado o Safety Culture: a Report by International Nuclear Safety Advisory Group (INSAG-4). Já no âmbito da saúde, a CSP é definida pela Agency for Healthcare Research and Quality (AHRQ) como valores, atitudes, percepções, competências e padrões de comportamento dos grupos e de pessoas. Isso determina 0 compromisso, estilo e proficiência no manejo da segurança em saúde de uma instituição (ANDRADE LEL, et al., 2018).

Para que ocorra o processo de implementação da cultura de segurança, é necessário que haja um profissional de enfermagem líder, capaz de conduzir os seus liderados por meio da comunicação efetiva, da promoção do desenvolvimento profissional, através de capacitações, do incentivo às práticas seguras e da realização do feedback a partir da análise dos eventos (MOTA DS, 2018; NEGREIROS BF, et al., 2018; SILVA SC, 2018).

Destaca-se o papel do enfermeiro, o qual lidera a maior equipe que presta assistência à saúde, que possui habilidades gerenciais e é o profissional responsável por grande parte das tomadas de decisões relacionadas à qualidade da assistência à saúde. Além de ser o gestor do processo de cuidado, que tem o papel basilar de promover cuidados seguros durante todo o tempo em que o paciente permanecer na instituição de saúde (MOTA DS, 2018; NEGREIROS BF, et al., 2018; SILVA SC, 2018).

O processo de implementação das estratégias de segurança do paciente deve envolver todos os profissionais, direta ou indiretamente enredados na assistência à saúde. Nesse âmbito, a participação do enfermeiro é fundamental na construção e efetivação do cuidado seguro, pois esse profissional é munido de conhecimento técnico e científico, lidera o maior contingente de trabalhadores da instituição e é responsável pela prestação de cuidados ao paciente durante as 24 horas do dia (MOTA DS, 2018; NEGREIROS BF et al., 2018; SILVA SC, 2018). Assim, o gestor deverá proporcionar atividades que estimulem os colaboradores ao conhecimento dos benefícios da mudança e assim obter a adesão às ações propostas (MOTA DS, 2018; NEGREIROS BF, et al., 2018; SILVA SC, 2018).

O profissional de saúde é o executor da ação assistencial, controlador dos processos e responsável pelos resultados, e é evidente que há sua responsabilidade nas boas práticas (treinamento, experiência e trabalho 
em equipe) e ferramentas de gestão clínica. Não podemos esquecer que o usuário não recebe todas as informações sobre o processo, além das expectativas dos pacientes mudarem e o profissional não saber disso. A melhoria da comunicação é um desafio atual, para muitos de nós dedicados à gestão da saúde, para melhorar a comunicação interna em todos os níveis, de forma bidirecional, facilitando a disponibilidade dos recursos possíveis, favorece a diminuição de falhas ou a sua detecção precoce (MOTA DS, 2018; NEGREIROS BF, et al., 2018; SILVA SC, 2018).

\section{CONSIDERAÇÕES FINAIS}

Portanto, é primordial repensar sobre a segura do paciente, pois são os profissionais de enfermagem que realizam o cuidado em tempo integral do paciente, manipulando, muitas vezes, do que qualquer outro profissional, porém isso não onera de toda a equipe multidisciplinar de ter esses conhecimentos, para que se evite os incidentes, garantindo qualidade na assistência prestada ao paciente. Este trabalho permitiu que os autores pudessem mostrar que a cultura de segurança do paciente é essencial, que é necessário que haja uma equipe de enfermeiros gestores preparados para realizar treinamentos rotineiros não só para a equipe de enfermagem, mas para toda a equipe multidisciplinar em unidades hospitalares, evitando, assim, os incidentes.

\section{REFERÊNCIAS}

1. ANDRADE LEL, et al. Cultura de segurança do paciente em três hospitais brasileiros com diferentes tipos de gestão. Ciência e Saúde Coletiva, 2018; 23(1): 161-172.

2. ARAÚJO LMA. A gestão do atendimento aos pacientes em tratamento de tuberculose pulmonar: a situação em duas unidades hospitalares de Maceió/AL. Dissertação (Mestrado em Gestão de Empresas) - Escola de Ciências e Econômicas e das Organizações. Universidade Lusófona de Humanidades e Tecnologias, Lisboa, 2017 ; 88 p.

3. ARAÚJO MO. Dinâmica organizativa do acesso de usuários do PSF aos serviços e às práticas de saúde de média e alta complexidade - Programa de Pós-graduação em Saúde Coletiva. Universidade Estadual de Feira de Santana, Feira de Santana, 2014; $191 \mathrm{p}$.

4. BAMPI R, et al. Perspectivas da equipe de enfermagem sobre a segurança do paciente em unidade de emergência. Revista de Enfermagem UFPE On Line, 2017; 11(2): 584-590.

5. BEHRENS R. Segurança do paciente e os direitos do usuário. Revista Bioética, 2019; 27(2): 253-260.

6. BRANDÃO MGSA, et al. Gestão de riscos e segurança do paciente: mapeamento dos riscos de eventos adversos na emergência de um hospital de ensino. Revista de Administração em Saúde, 2018; 18(70): 1-13.

7. CALDAS BN. Implementação do Programa Nacional de Segurança do Paciente em hospitais públicos: uma avaliação qualitativa. Tese (Doutorado em Saúde Pública) - Escola Nacional de Saúde Pública Sergio Arouca. Fundação Oswaldo Cruz, Rio de Janeiro, 2017; 242 p.

8. CARVALHO LM. Protocolo multiprofissional de atenção às urgências e emergências para imigrantes nos serviços de saúde. Dissertação (Mestre em Saúde Coletiva) - Programa de Pós-graduação em Saúde Coletiva. Universidade Federal de Goiás, Goiânia, 2019; 125 p.

9. COSTA BHF. Cuidado especializado à pessoa em situação crítica e em fim de vida. Dissertação (Mestre em Enfermagem Médico-cirúrgica) - Escola de Enfermagem. Instituto de Ciências da Saúde, Porto, 2017; 297 p.

10. FERREIRA BEM, et al. Adesão dos profissionais de enfermagem as metas de segurança da OMS: uma revisão de literatura. Revista Eletrônica Acervo Enfermagem, 2021; 8:1-8.

11. FRANCISCATTO L, et al. Metas internacionais de segurança do paciente em hospital universitário. Revista HCPA, $2011 ; 31(4): 482-486$.

12. INSTITUTO BRASILEIRO PARA A SEGURANÇA DO PACIENTE. 2018. In: Seis metas internacionais para a segurança do paciente. Disponível em: https://www.segurancadopaciente.com.br/seguranca-e-gestao/6-metasinternacionais-de-seguranca-do-paciente/. Acesso em: 15 abr. 2021.

13. KOERICH C. Significando a complexidade das interações profissionais na gestão da educação permanente em saúde, segurança do paciente e qualidade em hospitais públicos. Tese (Doutorado em Educação e Trabalho em Saúde e Enfermagem) - Programa de Pós-graduação em Enfermagem. Universidade Federal de Santa Catarina, Florianópolis, 2019; $268 \mathrm{p}$.

14. MOTA DS. Coaching: nova forma de liderança pelo enfermeiro. Monografia (Bacharel em Enfermagem) - Graduação em Enfermagem. Faculdade de Educação e Meio Ambiente, Ariquemes, 2018; 51 p.

15. NEGREIROS BF, et al. Avaliação da cultura de segurança do paciente em Centro de Terapia Intensiva de Hospital terciário. Revistas Tendências da Enfermagem Profissional, 2018; 10(4): 12-20.

16. OLIVEIRA JLC, et al. Segurança do paciente: conhecimento entre residentes multiprofissionais. Einstein (São Paulo), 2017; 15(1): 50-57.

17. RORATO TJ, et al. Formação de enfermeiros líderes: revisão integrativa. Revista Científica de Enfermagem, 2021; 11(33): 350-359. 
18. ROSA ERS, et al. As metas internacionais de segurança do paciente na percepção de estudantes de enfermagem. Revista Rede de Cuidados em Saúde, 2017; 11(1): 1-3.

19. SILVA AT, et al. Assistência de enfermagem e o enfoque da segurança do paciente no cenário brasileiro. Saúde Debate, 2016; 40(111): 292-301.

20. SILVA FS. Segurança do paciente: concepção da equipe de enfermagem sobre a qualidade do cuidado. Monografia (Bacharel em Enfermagem) - Graduação em Enfermagem. Faculdade Maria Milza, Governador Mangabeira, 2018; $65 \mathrm{p}$.

21. SILVA RFA, NASCIMENTO MAL. Mobilização terapêutica como cuidado de enfermagem: evidência surgida na prática. Revista da Escola de Enfermagem da USP, 2012; 46(2): 413-419.

22. SILVA SC. Percepções das profissionais da saúde acerca da cultura de segurança do paciente na internação obstétrica. Tese (Doutorado em Cuidado em Enfermagem e Saúde) - Programa de Pós-graduação em Enfermagem. Universidade Federal do Rio Grande do Sul, Porto Alegre, 2018; 182 p.

23. SCHMITT MD, et al. Análise de tese e dissertações sobre gestão de riscos na área da saúde no Brasil. Revista Mineira de Enfermagem, 2020; 24: 1-7.

24. SIMAN AG, BRITO MJM. Mudanças na prática de enfermagem para melhorar a segurança do paciente. Revista Gaúcha de Enfermagem, 2016; 37: 1-9.

25. SOARES VEA. Aplicações de instrumentos para avaliação da segurança do paciente ambulatorial quanto ao uso de medicamentos. Dissertação (Mestrado em Administração - Gestão de Sistemas de Saúde) - Programa de Mestrado Profissional em Administração. Universidade Nove de Julho, São Paulo, 2019; 122 p.

26. SOUZA TH. Recepção do usuário no SUS: estratégias para o acesso à rede de urgência e emergência, na perspectiva do trabalhador. Dissertação (Mestrado Profissional em Saúde) - Programa de Pós-graduação Multidisciplinar em Saúde. Universidade Federal de Santa Catarina, Florianópolis, 2014; 154 p.

27. VIANA ALVES EA. Segurança do paciente: do erro à prevenção do risco. Cadernos Ibero-Americanos de Direito Sanitário, 2013; 2(2): 723-723. 